\title{
Influência dos ácidos graxos ômega-3 e vitamina D na depressão: uma breve revisão
}

\author{
Influence of omega 3 fatty acids and vitamin D in depression: a short review \\ Juliana Severo Rosa ${ }^{1}$, José Ribamar Saraiva Junior ${ }^{2}$, André Gonzales Real ${ }^{3}$, Laís Quevedo Siqueira ${ }^{4}$, \\ Cláudia Severo Rosa ${ }^{*}$ \\ ${ }^{1}$ Médica psiquiatra; ${ }^{2}$ Médico psiquiatra, Docente - UFFS; ${ }^{3}$ Médico Residente; ${ }^{4}$ Terapeura Ocupacional; ${ }^{5}$ Doutora em \\ Ciências dos Alimentos. Docente do Departamento de Tecnologia e Ciência dos Alimentos da UFSM.
}

\begin{abstract}
Resumo
Objetivo: descrever uma revisão da literatura sobre a importância dos ácidos graxos ômega-3 e da vitamina D na prevenção e tratamento da depressão. Metodologia: essa revisão foi feita no período de agosto, setembro de 2016, incluindo as publicações de 2000 a 2016. Procedeu-se a busca nas bases de dados Pubmed, Science Direct, Bireme e periódicos CAPES com a utilização dos descritores: "ômega-3", "docosahexaenoic acid", "DHA", "eicosapentaenoic acid", "EPA", "vitamin D" e "depression". Resultados: a literatura reporta vários estudos sobre a eficácia dos ácidos graxos ômega-3 no tratamento da depressão. No entanto, existe um número considerável de trabalhos que não demonstram eficácia dos ácidos graxos ômega-3 na depressão. Os fatores responsáveis pelos resultados variados são: modelos experimentais, diferenças de tamanho da amostra, diferenças biológicas e genéticas entre os doentes, a variabilidade ambiental, e variabilidade na resposta à ácidos graxos ômega-3. Em relação a vitamina $\mathrm{D}$, os resultados também são contraditórios, com evidências sugerindo que baixos níveis dessa vitamina podem estar associados a um maior risco de depressão. Conclusão: não é possível afirmar que consumir alimentos ricos em ômega-3 e vitamina $D$, juntamente com o uso de fármacos possam aliviar os sintomas depressivos.

Palavras-chave: Depressão. Ácidos graxos poli-insaturados. Suplementação. Vitamina D
\end{abstract}

\begin{abstract}
Objective: to describe a literature review on the importance of omega-3 fatty acids and vitamin $D$ in the depression prevention and treatment. Methodology: this review was done in August / September, 2016, including publications from 2000 to 2016. Pubmed, Science Direct, Bireme and Journals CAPES databases were searched using the descriptors: "omega-3", "Docosahexaenoic acid "," $D H A$ "," eicosapentaenoic acid "," EPA "', vitamin D" and "depression ". Results: the literature report several studies on the efficacy of omega-3 fatty acids in the depression treatment. However, there are a number of studies that don't demonstrate efficacy of omega-3 fatty acids in depression. The factors responsible for the varied results are: experimental models, differences in sample size, biological and genetics differences among patients, environmental variability, and variability in response to omega-3 fatty acids. There are many researches with contradictory results regarding vitamin $D$, with evidence suggesting that low levels of this vitamin may be associated with an increased risk of depression. Conclusion: it can't be affirm that consume foods rich in omega-3 and vitamin D along with the use of pharmaceuticals products may alleviate depressive symptoms.
\end{abstract}

Keywords: Depression. Polyunsaturated Fatty Acids. Supplementation. Vitamin D.

\section{INTRODUÇÃO}

A depressão é um dos maiores problemas de saúde pública no mundo (KRUIJSHAAR et al., 2005). Sua etiologia não é completamente conhecida, além dos fatores psicossociais, diferentes fatores biológicos e ambientais são considerados para desencadear a fisiopatologia (SAVEANU; NEMEROFF, 2012). Seus sintomas podem incluir sentimentos de tristeza, crises de choro, baixa autoestima, angústia, isolamento social, pessimismo, culpa e baixa capacidade de sentir prazer, podendo variar em relação à sua intensidade e duração (ROZENTHAL; LAKS;

Correspondente/Corresponding: *Claudia Severo Rosa - End: Universidade Federal de Santa Maria, Departamento de Tecnologia e Ciência dos Alimentos, Santa Maria-RS. - Tel: (55)3220-8254 - E-mail: claudiasr37@yahoo.com.br
ENGELHARDT, 2004). Estimativas mostram que a depressão atinge mais de 350 milhões de pessoas ao redor do mundo, estando classificadas em terceiro lugar dentre os distúrbios que contribuem para a carga global da doença em 2030, sendo o tratamento mais importante a utilização de fármacos (MATHERS; LONCAR, 2006; LI et al., 2016). Atualmente, existem no mercado mais de oito classes de antidepressivos, mas $75 \%$ dos pacientes depressivos não apresentam resposta (LI et al, 2016). A atividade física pode ser uma aliada no combate à depressão, auxiliando na coordenação e equilíbrio, redução da incapacidade funcional e dos pensamentos negativos, redução de estresse e da sintomatologia depressiva (GUERRA et al., 2008).

O papel da dieta na saúde mental é pouco conhecido entre os clínicos. No entanto, o impacto que a nutrição 
pode ter sobre o cérebro é evidente. $O$ cérebro trabalha comandando a ingestão de nutrientes do corpo (BEYER; PAYNE, 2016). Conhecimentos nutricionais da psiquiatria mostram que a dieta pode contribuir para resistência ou risco na doença mental. Durante a última década, tem havido um número crescente de estudos bem delineados que têm demonstrado a associação de uma dieta saudável com melhorias em doenças e sintomas mentais, tais como a depressão, a ansiedade, a atenção, e irritabilidade (JACKA et al., 2012). Os efeitos positivos sugerem que certas intervenções dietéticas podem ser clinicamente relevantes para os pacientes, embora até o momento, não há dados disponíveis sobre o impacto terapêutico de mudanças na dieta sobre a depressão (O'NEIL et al., 2013).

Essas mudanças são refletidas principalmente no aumento de consumo de gordura animal e no desequilíbrio dos ácidos graxos ômega-3 e ômega-6 (ANDRADE; CARMO, 2006). Investigações clínicas têm sido realizadas a respeito do metabolismo dos ácidos graxos poli-insaturados, em particular com relação ao w-3. 0 consumo de ácidos graxos $w-3$ é muito baixo atualmente, porque diminuiu o consumo de peixes e principalmente porque cresceu a produção de alimentos ricos em w- 6 e pobres em w-3 (SIMOPOULOS; LEAF; SALEM, 2000).

Evidências mostram que mudanças na dieta podem melhorar a depressão (MANOSSO; MORETTI; RODRIGUES, 2013). A associação entre ácidos graxos w-3 e transtornos psiquiátricos, como a depressão, tem sido de grande importância (FENTON; HIBBELN; KNABLE, 2000). Alguns estudos mostram os benefícios da dieta mediterrânea (consumo de frutas, nozes e legumes), outros mostram os benefícios do consumo da carne e vegetais sobre a depressão (MEYER et al., 2013) e outros os benefícios da vitamina D (OKEREKE et al., 2016). Este estudo teve como objetivo revisar na literatura a importância do w-3 e vitamina $D$ na prevenção e tratamento da depressão.

\section{METODOLOGIA}

Para a elaboração desta revisão narrativa as seguintes etapas foram percorridas: definição da questão de pesquisa e dos objetivos do estudo; estabelecimento de critérios de inclusão; definição das informações a serem extraídas dos artigos selecionados; análise dos resultados; e apresentação dos resultados. Essa revisão foi feita no período de agosto, setembro de 2016, incluindo as publicações de 2000 a 2016. Procedeu-se a essa busca nas bases de dados Pubmed, Science Direct, Bireme e Periódicos CAPES, com a utilização dos descritores: "ômega-3", "docosahexaenoic acid", "DHA", "eicosapentaenoic acid", "EPA", "vitamin D" "depression", "depressão", "vitamina D". A estratégia de busca dos descritores foi combinada, utilizando-se "and" entre cada descritor. A busca foi realizada pelo acesso on-line, foram encontradas na primeira busca 654 pesquisas. Após leitura dos títulos restaram 171 pesquisas de interesse. Na leitura crítica dos resumos restaram 63 artigos. Adotaram-se os seguintes critérios de inclusão: artigos de revisão sistemática ou meta-análise, ensaios clínicos que utilizaram suplementação de ômega 3 ou vitamina $\mathrm{D}$ em transtornos neurológicos. Foram utilizados também livros específicos em bioquímica.

\section{Ácidos graxos poliinsaturados}

Entre os ácidos graxos poli-insaturados (PUFAs), destacam-se duas famílias, w-3 e 6, cada uma representada por um ácido graxo essencial, sendo o alfa-linolênico $(w-3)$ e o linoléico (w-6). Estes são conhecidos como essenciais, pois os seres humanos não podem sintetizá-los, portanto, precisam obtê-los a partir da ingestão dietética (JUMP, 2002).

Os ácidos graxos das séries ômega-3 e 6 representados pelos ácidos graxos essenciais alfa-linolênico e linoléico, respectivamente, contém duas ou mais insaturações e assim, são designados como poliinsaturados e são representados utilizando-se a seguinte simbologia numérica: C18:3 $(9,12,15)$ e C18:2 $(9,12)$. 0 ácido alfa-linolênico (ALA) pode ser convertido em ácido eicosapentanóico (EPA) e ácido decosahexanóico (DHA). $O$ ácido linoléico (LA), por sua vez, converte-se em ácido araquidônico (AA). Os ácidos graxos poli-insaturados AA, EPA e DHA, produtos da conversão dos essenciais, ALA e LA, por sua vez, convertem-se em metabólitos com efeitos biológicos distintos, conforme Figura 1 (PALANCA et al., 2006; JUMP, 2002).

Figura 1 - Conversão dos ácidos graxos essenciais das series ômega-3 e 6 em seus metabólitos

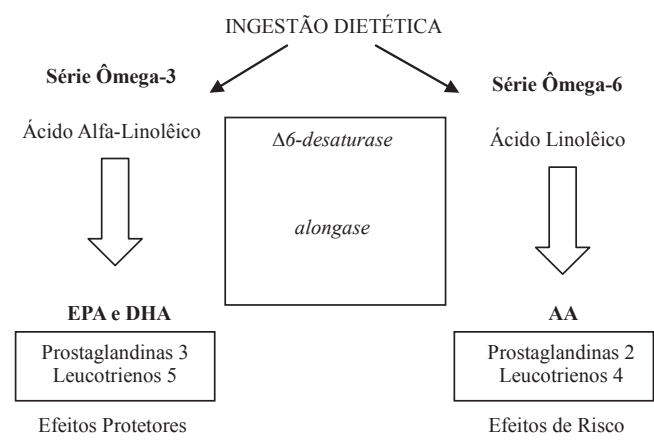

EPA: Ácido Eicosapentanóico

DHA: Ácido Decosahexanóico

AA: Ácido Araquidônico

Fonte: Barbosa et al. (2007)

O ácido alfa-linolênico, representante da família w-3, se converte nos ácidos eicosapenatanóico (EPA) e decosaehexanóico (DHA), que são precursores dos mediadores químicos, denominados prostaglandinas da série 3 e leucotrienos da série 5 , que desempenham efeitos biológicos protetores, enquanto que o ácido linoleico, representante da família do $w$ - 6 é convertido em ácido araquidônico, que é precursor da síntese dos eicosanoides que desempenham efeitos biológicos nocivos a saúde (BARBOSA et al., 2007). 
Existe uma competição entre os ácidos graxos w-3 e w-6 pelas mesmas enzimas de dessaturação ( $\Delta 6$ dessaturase) sendo que essas preferem o w-3 em detrimento ao w-6. Assim, os ácidos graxos EPA e DHA, produtos da conversão do ácido graxo alfa-linolenico (w-3) bloqueiam a ação da enzima $\Delta 6$ dessaturase inibindo a conversão do w- 6 em ácido araquidônico e, consequentemente a produção de eicosanoides da série par, prostaglandinas e leucotrienos 4. Dessa forma, os ácidos graxos w-3 exercem um efeito protetor, impedindo os eicosanoides da série par de exercer seus efeitos nocivos (BARBOSA et al., 2007).

\section{Ômega-3 e depressão}

Ao longo das últimas décadas tem sido relatado um aumento na incidência de depressão no mundo ocidental (SWEETING et al., 2010). No mesmo período, a dieta ocidental mudou significativamente, com uma enorme queda no consumo de w-3 em favor de um aumento na relação w6/w3 de 15-20:1, o que contrasta fortemente com a proporção ideal de cerca de $2: 1$ recomendado por especialistas da área de gorduras. Existem fortes indícios de que as alterações na proporção de ácidos graxos estão envolvidas na patogênese da depressão (SIMOPOULOS, 2009). Estudos epidemiológicos mostram que em países onde há maior consumo de peixes, que são fontes de w-3, os índices de depressão são menores (MEYER et al., 2013; SILVA et al., 2013). Várias pesquisas demonstraram que pacientes deprimidos em geral, têm significativamente menor quantidade de w-3 nos fosfolípidios, tanto na membrana de eritrócitos como livre no plasma. O possível mecanismo dos ácidos graxos poli-insaturados (w-3) na depressão é a proporção destes ácidos na membrana das células, os quais dependem da dieta. Uma dieta com baixa relação $w-6$ para $w-3$ (ideal) fará a dessaturação competitiva e a incorporação nos fosfolípidios de uma quantidade elevada de EPA e DHA na membrana da célula, e consequentemente níveis inferiores de ácido araquidônico, que em quantidades elevadas são ruins (HUSTED; BOUZINOVA, 2016).

Os transtornos de humor têm sido relacionados com o consumo de ácidos graxos poli-insaturados, pois os lipídios são essenciais para a função e estrutura normal do cérebro. A elevada razão entre ácidos graxos da série w-6/w-3 têm sido relacionados com o aumento da incidência de desordens psiquiátricas (SIMOPOULOS, 2009). Essa mudança na dieta é considerada um fator relevante na deficiência em fosfolipídios (YOUNG; MARTIN, 2003). Fosfolipídios compostos por w- 6 e w-3 têm uma importante função na tradução dos sinais nervosos, na integridade da membrana celular e na sua fluidez. O balanço correto desses ácidos graxos nos fosfolipídios é essencial para o funcionamento neural normal, o rompimento desse balanço pode desencadear depressão (BARBOSA et al., 2007). Beyer e Payne (2016) têm sugerido que o EPA é o responsável pelos efeitos benéficos dos ácidos graxos $w-3$ em doenças depressivas, e que os suplementos contendo mais de $60 \%$ de EPA são mais eficazes contra depressão primária. Esta informação é surpreendente dado que o DHA é um importante componente estrutural das membranas neuronais e o EPA está presente em níveis relativamente muito mais baixos. Há várias explicações possíveis para esta evidência, incluindo o fato de que o DHA é pouco incorporado no cérebro humano e o EPA pode facilitar este processo, outra explicação pode ser o efeito anti-inflamatório dos eicosanóides EPA-derivados, a sua eficácia reduzindo as citocinas inflamatórias, fator de necrose tumoral-a, interleucina (IL) - 6 e IL-1b, e aumentando a $\mathrm{N}$-acetil-aspartato no cérebro (sugerindo um papel neuroprotector). Além disso, a evidência atual suporta apenas o seu uso como um tratamento adjunto, não constitui uma alternativa para psicofarmacologia padrão, entretanto, o w-3 não parece ter quaisquer efeitos adversos significativos e pode ser importante para saúde em geral (MONTGOMERY; RICHARDSON, 2008; BEYER; PAYNE, 2016).

Evidências ao longo das últimas três décadas sugerem que a dieta pobre em $w$-3, especialmente durante o desenvolvimento perinatal, pode representar um fator de risco para transtornos depressivos. Estudos transversais mostram evidências que adultos e pacientes com transtornos depressivos apresentam déficits de ácidos graxos $\mathrm{w}-3$ no sangue comparado com indivíduos saudáveis (WANI; BHAT; ARA, 2015). A suplementação da dieta com óleo de peixe é suficiente para corrigir a falta no sangue de w-3 em pacientes com transtornos depressivos e ensaios controlados sugerem que a suplementação pode reduzir os sintomas depressivos. Embora controversa, a evidência sugere também que a deficiência em w-3 pode aumentar o risco de suicídio e doenças cardiovasculares, duas principais causas do excesso de mortalidade prematura em pacientes com transtornos depressivos (MCNAMARA, 2016). No entanto, existem vários estudos que não mostram nenhuma eficácia dos ácidos graxos $\mathrm{w}-3$ na depressão. Isto pode ser explicado por fatores que podem ser responsáveis pelos diferentes resultados, tais como, modelos experimentais de variáveis, diferenças no tamanho da amostra, diferenças biológicas e genéticas entre os pacientes e variabilidade na resposta ao w-3 (BOS et al., 2016). A depressão é uma doença multifatorial, e os pacientes que têm depressão por falta de $\mathrm{w}-3$ podem reagir bem à dieta contendo altos níveis deste ácido, podendo mostrar sinais positivos em relação ao tratamento (BOS et al., 2016). Contudo, para os pacientes que sofrem de depressão por outros fatores além do w-3, podem não ter uma resposta adequada. Esta poderia ser a razão pela qual a literatura mostra resultados conflitantes sobre a eficácia do w-3. Para chegar à conclusão sobre a eficácia dos ácidos graxos w-3 no tratamento da depressão, é necessário categorizar os pacientes de depressão com base nas suas causas, contudo, considerando as variações individuais da depressão e resposta a certas estratégias de tratamento ajudaria a chegar a uma conclusão mais clara (WANI; BHAT; ARA, 2015). 
Uma revisão feita por Freeman et al. (2006) recomenda indivíduos deprimidos a tomar com segurança 1 g da mistura de EPA / DHA, mas que essa mistura de w-3 não deve substituir os antidepressivos convencionais, do mesmo modo Mischoulon e Freeman (2013) afirmam que indivíduos que tomam mais de $3 \mathrm{~g}$ de $\mathrm{w}$-3 por dia devem fazê-lo sob supervisão médica. Não foram encontrados estudos mostrando que o consumo de alimentos ricos em w-3 possa atuar na melhora do quadro depressivo, entretanto existem vários alimentos ricos em w-3 como, peixes de água salgada (salmão, sardinha, atum, cavala) ou o óleo de peixe, que apresentam maior quantidade de ácidos graxos w-3 (HARRIS, 2001). Os principais ácidos graxos w-3 são o ácido linolênico 18:3, o EPA (20:5) e o DHA (22:6).

A semente de chia (Salvia hispânica) é nativa da região que se estende do oeste central do México ao norte da Guatemala. Contém de 40 a $53 \%$ de óleo, nos quais os ácidos graxos poli-insaturados $\alpha$-linolênico ( $w$-3) e linoléico ( $w-6)$ estão presentes em quantidades elevadas (60 e 30\%, respectivamente) (IXTAINA et al., 2011), sendo estas quantidades significativamente maiores que nos óleos de soja, linhaça e canola (GALLI; MARANGONI, 2006). Embora a chia não seja um alimento muito conhecido, sua produção global está aumentando devido às suas propriedades saudáveis. O óleo de chia contém umas das maiores concentrações conhecida de ácido $\alpha$-linolênico e em função disto, tornou-se uma importante opção para a nutrição pelos benéficos efeitos para a saúde humana, como a proteção contra eventos cardiovasculares (MOZZAFFARIAN, 2005). As sementes de chia são também utilizadas como suplementos alimentares, bem como na fabricação de barras de cereais, cereais matinais e biscoitos nos EUA, América Latina, Austrália e Brasil (DUNN, 2010).

Perilla (Perilla frutescens) é uma planta nativa dos países asiáticos usada na culinária e na medicina tradicional. No entanto, é desconhecida para as populações ocidentais (KUROWSKA et al., 2003). Suas sementes contêm aproximadamente $35-45 \%$ de óleo, sendo $64 \%$ de (w-3). O óleo de perilla constitui aproximadamente $40 \%$ do peso da semente, ainda é composto por ácido palmítico (C16: 0), ácido esteárico (C18: 0), ácido oléico (C18: 1n-9) e ácido linoléico (C18: 2n-6) (LEE et al., 2002). A ingestão de óleo de perilla contendo grande quantidade de ácido graxo $\alpha$-linolênico oferece vários benefícios à saúde, como a redução do nível de lipídios no plasma (KIM et al., 2004).

A linhaça (Linum asitatissimun) é geralmente encontrada como grão integral, moído, ou na forma de óleo. Atribui-se a linhaça, o sabor e o aroma de nozes, podendo ser facilmente incorporada a diversos produtos, tanto integralmente, como moída, apresenta de 32,3 a 41\% de óleo, sendo que $57 \%$ desse óleo é w-3, 16\% de w-6, $18 \%$ de ácido graxo monoinsaturado. A predominância de $w-3$ é três vezes superior a w-6. Alguns exemplos de produtos são pães, biscoitos, bolos tipo muffins, biscoitos tipo cookies e bolos (MORRIS, 2001).
As nozes verdadeiras são frutas secas, espessas e muitas vezes contêm espinhos que recobrem sua semente. As mais conhecidas são: amêndoa, noz pecã, castanha-do-pará, castanha-de--caju, pistache, avelã, macadâmia e castanha. Além das nozes verdadeiras, existem muitas sementes comestíveis com características semelhantes a elas, mas com classificação botânica diferente. É o caso do amendoim, a amêndoa de baru (Dipteryx alata), proveniente do fruto do barueiro, leguminosa arbórea lenhosa nativa do Cerrado. As nozes verdadeiras e as sementes comestíveis, como o amendoim e a amêndoa de baru, contêm teores elevados de lipídeos (cerca de $40 \%$ a $60 \%$ ). Dentre as nozes e sementes comestíveis, a macadâmia possui a melhor proporção de ácidos graxos $\omega-6 / \omega-3$, seguida pela noz, castanha e amêndoa de baru (FREITAS; NAVES, 2010).

Evidências epidemiológicas atuais sugerem que o consumo de uma dieta rica em peixes, frutas, legumes, e grãos integrais podem reduzir o risco de depressão. No entanto, esses resultados têm sido um pouco inconsistentes. Isto pode ser porque muitas pesquisas que estudam dieta e depressão não tem o conhecimento sobre as associações de nutrientes à depressão ou possíveis mecanismos que liguem a relação dieta/depressão e consequentemente não podem diagnosticar a dieta mais relevante (LAI et al, 2016).

\section{Vitamina D e depressão}

Os níveis de vitamina $\mathrm{D}$ dependem da exposição solar, pigmentação da pele, tipo de vestuário, nutrição e ingestão de suplementos Van Schoor e Lips (2011), sendo que a dieta se torna mais importante à medida que a exposição solar diminui. É possível determinar os níveis de vitamina $D$ através da dosagem sérica de 25-hidroxicolecalciferol (25(OH)D3). Embora não haja um consenso sobre a quantidade ideal de vitamina $\mathrm{D}$, a maioria dos pesquisadores concorda que a dosagem sérica de 25(OH)D3 deve ser maior que $50 \mathrm{nmol} / \mathrm{L}$, embora também haja recomendação de níveis maiores, como 75 ou $100 \mathrm{nmol} / \mathrm{L}$ (JU et al., 2013). Considera-se deficiência de vitamina $D$ quando a dosagem sérica se apresenta em níveis inferiores a 25 nmol/L (ESERIAN, 2013; VAN SCHOOR; LIPS, 2011). Atualmente, a maioria dos autores adota valores entre 25 e $50 \mathrm{nmol} / \mathrm{L}$ (10 a $20 \mathrm{ng} / \mathrm{mL}$ ) para deficiência moderada e inferiores a $25 \mathrm{nmol} / \mathrm{L}(10 \mathrm{ng} / \mathrm{mL})$ para deficiência grave (PREMAOR; FURLANETTO, 2006).

Hipóteses recentes sobre os mecanismos fisiopatológicos na associação entre vitamina D e depressão incluem a vitamina $D$ na regulação dos neurotransmissores, dopamina, noradrenalina e acetilcolina, bem como um efeito de fatores neurotróficos (HUMBLE, 2010). Além disso, receptores de vitamina $D$ são encontrados no córtex pré-frontal e peças do sistema límbico (PRICE; DREVETS, 2010). Estas áreas do cérebro têm sido implicadas na fisiopatologia da depressão. A vitamina $D$ também pode reduzir concentrações de marcadores inflamatórios as- 
sociados com a depressão (EYLES et al., 2005; VAN DEN BERG et al., 2016) A diminuição da exposição ao sol, a ingestão dietética mais pobre e o tabagismo, pode fazer com que a deficiência de vitamina $\mathrm{D}$, leve a depressão (VAN DEN BERG et al., 2016).

É cada vez mais evidente que a deficiência de vitamina $D$ está sendo sugerida como um contribuinte para depressão (MILANESCHI, et al., 2014). A presença de receptores de vitamina $D$ e as enzimas de ativação da vitamina $D$ em várias partes do cérebro humano contribuem para a regulação do humor e depressão, como o hipocampo, hipotálamo e córtex pré-frontal o que traz explicações biológicas plausíveis para a relação entre a deficiência de vitamina D e depressão (ANGLIN et al., 2013, RABENBERG, et al., 2016). Leedahl et al.( 2013) verificaram que indivíduos que recebiam suplementação com vitamina D na dose de 400 ou 600 U.I./dia durante um período de seis meses relataram melhora na sensação de bem-estar com a dose mais alta. Outro estudo onde indivíduos receberam placebo ou suplementação com vitamina D na dose de 20.000 ou 40.000 U.I./semana durante um período de um ano, observou-se que indivíduos suplementados apresentaram diminuição significativa na pontuação do Inventário de Beck para Depressão, instrumento utilizado para avaliar a intensidade da doença, no qual um maior número de pontos equivale à intensidade mais grave (LEEDAHL et al., 2013). Entretanto em idosas suplementadas com vitamina D na dose de 500.000 U.I./ ano durante um período de três a cinco anos, não se observou associação entre a suplementação com vitamina D e depressão (ESERIAN, 2013; SANDERS et al., 2011). Resultados discrepantes entre os estudos podem ser explicados através da diferença das doses de vitamina $D$ utilizadas nos mesmos. Embora não se saiba qual a dose ideal de vitamina $\mathrm{D}$ para o tratamento de sintomas depressivos, resultados positivos parecem estar correlacionados a doses mais altas (ESERIAN, 2013; LEEDAHL et al., 2013).

Recentemente, Toffanello et al.(2014) relataram que a atividade física parece modificar a associação de vitamina $D$ com depressão, entretanto verificaram uma associação indireta entre vitamina $D$ e depressão, onde a atividade física pode ser vista como um mediador. Johansson et al. (2016) verificaram a associação em pacientes com níveis de vitamina $D$ abaixo de $50 \mathrm{nmol} / \mathrm{l}$. Um estudo semelhante conduzido por Jorde e Grimnes (2015), verificou que pacientes com níveis de vitamina $D$ abaixo de $50 \mathrm{nmol} /$ I, devem ter suplementação de vitamina D.

Gowda et al. (2015) fizeram um estudo de meta-análise de ensaios clínicos randomizados com indivíduos com depressão para estimar o efeito da suplementação de vitamina D. Foram pesquisados artigos de 2013 e 2014 num total de 4923 participantes. Nenhuma redução significativa na depressão foi observada após a suplementação de vitamina $\mathrm{D}$, no entanto, à maioria dos estudos estavam focados em indivíduos com baixos níveis de depressão e suficientes níveis séricos de vitamina D. Futuros ensaios clínicos randomizados que incluam indivíduos deprimidos com baixos níveis séricos de vitamina $D$ são necessários para determinar a eficácia da vitamina $D$ no tratamento da depressão.

Os sintomas depressivos são comuns durante o inverno entre os países de latitudes do norte, quando os níveis de Vitamina $D$ são baixos pela quantidade inadequada de radiação ultravioleta (UVB), resultando em diminuição da síntese de vitamina D na pele. Entretanto existem poucos estudos sobre a influência da estação do ano sobre a associação de vitamina $D$ e depressão (BRANDENBARG, et al., 2012; HOANG et al., 2011). Três estudos não observaram a influência da estação (BRADENBARG, et al., 2012; NANRI et al., 2009; STEWART; HIRANI, 2010) enquanto outros dois relataram evidências conflitantes e uma associação inversa entre a vitamina $D$ e sintomas depressivos no inverno Noang et al. (2011) e no verão (KJAERGAARD; JOAKIMSEN; JORDE, 2011).

Rabenberg et al. (2016) estudaram a relação estação do ano: vitamina $D$ e depressão através do Patient Health Questionnaire (PHQ-9) (versão alemã do módulo de depressão 9-item do questionário de saúde do paciente) e verificaram que os níveis de depressão são maiores no verão e não no inverno na população entre 18-79 anos na Alemanha. A associação no verão sugere que a relação entre os níveis de vitamina $D$ e os sintomas depressivos são maiores quando a exposição à radiação UVB são mais elevadas. Estes resultados mostram a ideia de que a deficiência de vitamina $D$ pode ser uma consequência e não a causa da depressão. Da mesma forma do w-3 não foram encontrados artigos que mostrem a eficiência dos alimentos ricos em vitamina $D$ nos quadros depressivos, entretanto existem muitos alimentos ricos em vitamina $D$ que se consumidos como adjuvantes a medicação possam melhorar o quadro depressivo.

Apesar da vitamina D ser produzida por exposição da pele aos raios solares, seu consumo dietético se torna essencial quando a exposição solar é insuficiente para alcançar as necessidades diárias. Entretanto, são escassos os alimentos fonte de vitamina $D$, como a gema de ovo, fígado, manteiga e leite. Alguns fatores dietéticos têm sido apontados como auxiliares ou redutores da biodisponibilidade da vitamina $D$. Porém, sabe-se que o leite ingerido conjuntamente com fontes naturais de vitamina $D$ pode elevar de três a 10 vezes sua absorção, pela presença da lactoalbumina. Ácidos graxos de cadeia longa também facilitam a absorção de vitamina D (BUENO; CZEPIELEWSKI, 2008).

\section{CONCLUSÃO}

A literatura traz vários estudos sobre a eficácia dos ácidos graxos ômega-3 no tratamento da depressão. No entanto, existe um número considerável de estudos que não demonstram eficácia dos ácidos graxos ômega-3 na depressão. Os fatores responsáveis pelos resultados variados são: modelos experimentais, diferenças de tamanho da amostra, diferenças biológicas e genéticas entre os 
doentes, a variabilidade ambiental, e variabilidade na resposta à ácidos graxos ômega-3. Os resultados sobre a associação entre vitamina $D$ e depressão são contraditórios, sugerindo que baixos níveis dessa vitamina podem estar associados ao maior risco de depressão. Não é possível afirmar que consumir alimentos ricos em ômega-3 e vitamina $D$, juntamente com o uso de fármacos possa aliviar os sintomas depressivos.

\section{REFERÊNCIAS}

ANDRADE, P. M.; CARMO, M. Ácidos graxos ômega 3: um link entre eicosanoides, inflamação e imunidade. Metab., Lavras, v. 8, n. 3, p. 135-143, 2006.

ANGLIN, R. E. et al. Vitamin D deficiency and depression in adults: systematic review and meta-analysis. Braz. J. Psych., Rio de Janeiro, v. 202, p. 100-107, 2013.

BARBOSA, K. B. F. et al. Ácidos graxos da série ômega 3 e 6 e suas implicações na saúde humana. Rev. Nutrire, São Paulo, v. 32, n.2, p. 129-145, 2007.

BEYER, J.; PAYNE, M. E. Nutrition and bipolar depression. Psych. Clin. North Am., Filadélfia, v. 39, p. 75-86, 2016.

BOS, D. et al. Effects of omega-3 polyunsaturated fatty acids on human brain morphology and function: What is the evidence? Eur. Neuropsy., Londres, v. 26, p. 546-561, 2016.

BUENO AL; CZEPIELEWSKI, MA. The importance for growth of dietary intake of calcium and vitamin D. J. Pediatr., Rio de Janeiro, v. 84, n.5, p. 386-394, 2008.

BRADENBARG J. et al. Maternal early-pregnacy vitamin D status is associated with maternal depressive symptoms in the Amsterdam born children and their development cohort. Psych. Medic., Nova lorque, v. 74, p. 751-757, 2012

DUNN, J., 2010. The chia company seeks entry into European market. Disponivel em: <http://www.ausfoodnews.com.au/2010/02/08/the-chia-companyseeks-entry-into-european-market.html>.Acesso em: 2 June 2016.

ESERIAN, J. K. Papel da vitamina D no estabelecimento e tratamento de transtornos neuropsiquiátricos. Rev. Ciên. Méd. e Biol., Salvador, v.12, n. 2, p. 234-238, 2013.

EYLES, D. et al. Distribution of the vitamin D receptor and 1 alpha-hydroxylase in human brain. J. Chem. Neuroanatomic, Amsterdam, v. 29, n. 1, p. 21-30, 2005.

FENTON, W. S.; HIBBELN, J.; KNABLE, M Essential fatty acids membrane abnormalities, and the diagnosis and treatment of schizophrenia. Biol. Psych., Oxford, v. 47, p. 8-21, 2000.

FREEMAN, M. P. et al. Omega-3 fatty acids: evidence basis for treatment and future research in psychiatry. J. Clin. Psych., Nova lorque, v. 67, p.1954-1967, 2006

FREITAS, J.; NAVES, M. Composição química de nozes e sementes comestíveis e sua relação com a nutrição e saúde. R. Nutr., Campinas, v. 23, n. 2, p. 269-279, 2010.

GOWDA, U. et al. Vitamin D supplementation to reduce depression in adults: meta-analysis of randomized controlled trials. Nutr., Edimburgo, v. 32, p. 421-429, 2015

GUERRA, A. et al. Efeitos do consumo ou suplementação de omega 3 e do exercicio físico na prevenção e tratamento da depressão. Rev. Bras. Nutr. Esport., São Paulo, v. 2, n. 9, p. 145-153, 2008.
GALLI, C.; MARANGONI, F. N-3 fatty acids in the Mediterranean diet. Prost. Leuk. Essen. Fatty Acids, Nova lorque, v. 75, n. 3, p. 129-133, 2006.

HARRIS, W. S. Nonpharmacologic treatment of hypertriglyceridemia: focus on fish oils. Clin. Cardiology, Besterda, v. 22, supl. 6, p. 40-43, 2001.

HOANG, M. et al. Association between low sérum 25-hydroxyvitamin D and depression in a large sample of healthy adults: the cooper center longitudinal study. Mayo Clinic Proc., Chicago, v. 86, p. 1050-1055, 2011.

HUMBLE, M. Vitamin D, light and mental health. J. Photochem. Photobiol. B., Toronto, v. 101, n. 2, p.142-149, 2010.

HUSTED, K.; BOUZINOVA, E. The importance of $n-6 / n-3$ fatty acids in the major depressive disorder. Medic., Washington, v. 133, p. 1-9, 2016.

IXTAINA, V. Y. et al. Characterization of chia seed oils obtained by pressing and solvent extraction. J. Food Comp. Anal., Washington, v. 24, n. 2, p. $166-174,2011$.

JACKA, F. N. et al. Nutrient intakes and the common mental disorders in women. J. Affec. Dis., Nova lorque, v. 141, n. 1, p. 79-85, 2012.

JOHANSSON, P. et al. Vitamin D levels and depressive symptoms in patients with chonic heart failure. Inter. J. Cardio., Londres, v. 207, p. 185-189, 2016.

JORDE, R.; GRIMMES, G. Vitamin D and health: the need for more randomized controlled trials. J. Steroid Biochem. Mol. Biology, Malden, v. 148, p. 269-274, 2015.

JU, S.; LEE, Y.; JEONG, S. Serum 25-hydroxyvitamin D levels and the risk of depression: a systematic review and meta-analysis. J. Nutr. Health Aging, Toronto, v. 17, n.5, p. 447-455, 2013.

JUMP, D. B. The biochemistry of n-3 polynsatured fatty acids. J. Biol. Chem., Edimburgo, v. 277, n. 11, p. 8755-8758, 2002.

$\mathrm{KIM}, \mathrm{H}$. K.; $\mathrm{CHOI}$, S.; $\mathrm{CHOI}$, H. Suppression of hepatic fatty acid synthase by feeding $\alpha$-linolenic acid rich perilla oil lowers plasma triacylglycerol level in rats. J. Nutri. Biochem., Amsterdam, v. 15, n. 8, p. 485-492, 2004.

KJAERGAARD, M.; JOAKIMSEN, R.; JORDE, R. Low sérum 25-hydroxyvitamin $D$ levels are associated with depression in adult Norwegian population. Psych. Res., Amsterdam, v. 190, n. 2/3, p. 221-225, 2011.

KUROWSKA, E. et al. Bioavailability of omega-3essential fatty acids from perilla seed oil. Prost. Leuk. Essen. Fatty Acids, Washington, v. 68, n.3, p. 207-212, 2003.

KRUIJSHAAR M. E. et al. Life time prevalence estimates of major depression: an indirect estimation method and quantification of recall bias. Eur. J. Epidemiol., Besterda, v. 11, p.103-111, 2005

LAI, J. S et al. Inflammation mediates the association between fatty acid intake and depression in older men and women. Nutr. Res., Besterda, v. 36, n. 3, p. 234-245, 2016.

LEEDAHL, D. D. et al. Hypovitaminosis D in Psychiatric Inpatients: Clinical Correlation with Depressive Symptoms, Cognitive Impairment, and Prescribing Practices. Psych., Amsterdam, v. 54, n. 3, p. 257-262, 2013.

LEE, B.H.; RYU, S.N.; KWAK, T.S. Current status and prospects of quality evaluation in perilla. Korean J. Crop Sci., Koreia, v. 47, p. 150-162, 2002.

LI, J. et al. Tea consumption is inversely associated with depressive symptoms in the elderly: a cross-sectional study in eastern China. J. Affect. Dis., Nova lorque, v. 99, p. 157-162, 2016.

MANOSSO, L.; MORETTI, M.; RODRIGUES, A. Nutritional strategies for dealing with depression. Food Funct., Buenos Aires, v. 4, n. 12, p. 1776-1793, 2013. 
MATHERS, C. D.; LONCAR, D. Projections of global mortality and burden of disease from 2002 to 2030. PLoS Med, São Francisco, v. 3, n.11, p. e 442, 2006.

MCNAMARA, R. Role of omega-3 fatty acids in the etiology, treatment, and prevention of depression:current status and future directions. J. Nutr. Intermediary Metabolism, Nova lorque, v. 5, p. 96-106, 2016.

MEYER, B. et al. Food groups and fatty acids associated with self-reported depression: An analysis from the Australian National Nutrition and Health Surveys. Nutr., Edimburgo, v. 29, p.1042-1047, 2013.

MILANESCHI, Y. et al. The association between low vitamin $D$ and depressive disorders. Mo. Psych., Oxford, v. 19, n. 4, p. 444-451, 2014.

MISCHOULON, D. E.; FREEMAN, M. Omega-3 fatty acids in psychiatry. Psych. Clin. North Am., Filadélfia, v. 36, n.1, p. 15-23, 2013.

MONTGOMERY P.; RICHARDSON, A. J. Omega-3 fatty acids for bipolar disorder (review). Cochrane Database Syst Rev., Oxford, n.2, CD005169, 2008.

MORRIS, D. H. Essencial nutrients and other functional compounds in flaxseed. Nutr. Today, Besterda, v. 33, n.3, p.159, 2001.

MOZZAFFARIAN, D. Does alpha-linolênic acid intake reduce the risk of coronary heart disease? A review of the evidence. Alt. Therapies Health Med.,Toronto, v. 11, n. 3, p. 24-30, 2005.

NANRI, A. et al. Association between sérum 25-hydroxyvitamin D and depressive symptoms in japanese: analysis by survey season. Eur. J. Clin. Nutr., Londres, v. 63, n. 12, p. 1444-1447, 2009.

O'NEIL, A. et al. A randomised, controlled trial of a dietary intervention for adults with major depression (the "SMILES" trial): study protocol. BMC Psych., Londres, v.13, p.114, 2013.

OKEREKE, O.; SINGH, A. The role of vitamin D in the prevention of late-life depression. J. Affec. Disor., Nova lorque, v. 198, p. 1-14, 2016.

PALANCA, V. et al. Bases científicas para el desarrollo de produtos carnicos funcionales com actividad biológica combinada. Nutr. Hosp., Buenos Aires, v. 21, n. 2, p. 199-202, 2006.

PRICE, J. L.; DREVETS, W. C. Neurocircuitry of mood disorders. Neuropsychopharmacol., Berlim, v. 35, n. 1, p. 192-216, 2010.

PREMAOR, M.; FURLANETTO, T. Hipovitaminose D em adultos: entendendo melhor a apresentação de uma velha doença. Revisão. Arq. Bras. Endocrinol. Metab., São Paulo, v. 50, n.1, 2006.

RABENBERG, $M$. et al. Association between vitamin $D$ and depressive symptoms varies by season: results from the German Health Interview and Examination Survey for Adults (DEGS1). J. Affec. Disor., Nova lorque, v. 204, p. 92-98, 2016.

ROZENTHAL, M.; LAKS, J.; ENGELHARDT, E. Aspectos neuropsicológicos da depressão. Rev. Psiq., Porto Alegre, v. 26, n. 2, p. 204-212, 2004.

SANDERS, K. M. et al. Annual high-dose vitamin D3 and mental well-being: randomised controlled trial. Brit. J. Psych., Londres, v. 198, n. 5, p. 357-364, 2011.

SAVEANU, R. V.; NEMEROFF, C. B. Etiology of depression: genetic and enviromental factors. Psych. Clinic N. Am., Filadélfia, v. 35, p. 51-71, 2012.

SILVA, M. et al. Ácidos graxos no tratamento de doenças cardíacas, esquizofrenia e depressão. Anais da mostra científica do CESUCA, v.1, n.7, p. 1-13, 2013.

SIMOPOULOS, A. P. Evolutionary aspects of the dietary ômega 6:ômega 3 fatty acid ratio: medical implications. World Review Nutr. Diet, Malden, v. 100, p. 1-21, 2009.

SIMOPOULOS, A. P.; LEAF, A.; SALEM, N. Workshop on the essentiality of and recommended dietary intakes for ômega 6 and ômega 3 fatty acid. J. Am. Coll Nutr., Chicago, v. 18, n. 5, p. 487-489, 2000.

STEWART, R.; HIRANI, V. Relationship between vitamin D levels and depressive symptoms in older residentes from a national survey population. Psych. Medic., Oxford, v. 72, n. 7, p. 608-612, 2010.

SWEETING, H. et al. Can we explain increases in young people's psychological distress over time. Soc. Science Medic., Nova lorque, v. 71, n.10, p. 1819-1830, 2010.

TOFFANELLO, E. D. et al. Serum 25-hydroxyvitamin D and the onset of late-life depressive mood in older men and women: the pro.V.A. study. J. Gerontol. A. Biol. Science Med. Science, Nova lorque, v. 69, p. 1554-1561, 2014.

VAN DEN BERG, K. et al. Vitamin D deficiency, depression course and mortality: longitudinal results from the Netherlands study on depression in older persons (NESDO). J. Psychomatic. Res., Malden, v. 83, p. 50-56, 2016.

VAN SCHOOR, N.M.; LIPS, P. Worldwide vitamin D status. Best. Pract. Res. Clin. Endocrinol. Metab., Chicago, v. 25, n. 4, p. 671-680, 2011.

WANI, A. L.; BHAT, S.; ARA, A. Omega-3 fatty acids and the treatment of depression: a review of scientific evidence review. Integr. Med. Res., Oxford, v. 4, n.3, p. 132-142, 2015.

YOUNG, C.; MARTIN, A. Omega-3 fatty acids in mood disorders: an overview. Rev. Bras. Psiquiatr., São Paulo, v. 25, n. 3, p. 184-187, 2003.

Submetido em: 15/09/2016

Aceito em: 24/04/2017 